\title{
Impact of the minimum inhibitory concentration on the efficacy of oxitetracycline in the treatment of streptococcus agalactiae in Nile Tilapia
}

\begin{abstract}
Streptococcosis is one of the main causes of morbidity and mortality in Brazilian tilapia farming and oxytetracycline is one of the main antibiotics that have been used for decades in its treatment. However, studies of experimental infections carried out in Brazil with Streptococcus agalactiae with MIC $0.24 \mathrm{ug} / \mathrm{mL}$ treated with oxytetracycline orally at a dose of $100 \mathrm{mg} / \mathrm{kg}$ every 12 hours showed $20 \%$ of mortality rates and $60 \%$ of carrier animals. The objective of the present study was to evaluate the effect of changes in Streptococcus agalactiae MIC isolated on mortality, carrier and cure rates using pharmacokinetic/pharmacodynamic (PK/PD) modeling. The index used for the determination of efficacy was the area under the plasma concentration versus time curve (AUC)/minimum inhibitory concentration (MIC). A Monte Carlo simulation of AUC followed by pharmacokinetic/pharmacodynamic modeling was used to estimate the rates of cured, carrier and dead animals. Changes in mortality rates and estimated asymptomatic carriers related to changes in the MIC were observed. The estimated mortality rates were $0.3 \% ; 2.5 \% ; 25 \%$ and $90 \%$ and carrier rates were $1.3 \% ; 16.7 \%$; $60 \%$ and $9 \%$ according to MICs of strains $0.06 ; 0.125 ; 0.25$ and $0.5 \mathrm{ug} / \mathrm{mL}$, respectively. In this simulation study it was observed that small increments in the MIC determined large changes in the rates of cured, runner and dead animals. This demonstrates the need to incorporate in the therapeutic protocol bacteriological isolation, determination of MIC and optimization of therapeutic doses in order to avoid therapeutic failures and consequently potentiate the development of resistance.
\end{abstract}

Keywords: pharmacodynamic, pharmacokinetic, streptococcosis, tetracycline, tilapiculture
Volume 6 Issue 5- 2018

\section{IrisFatima Mariotto, Ticiane Moreira da Silva Lima, Sheila Rezler Wosiacki, Marcos Ferrante}

Department of Veterinary Medicine, Maringa State University, Brazil

Correspondence: Marcos Ferrante, Department of Veterinary Medicine, Maringa State University, Brasil, Tel +55(44) 3621 9412, Email marcosferrante@gmail.com

Received: July 17, 2018 | Published: September 05, 2018

\section{Introduction}

Nile tilapia, Oerochromis niloticus, a species that has been undervalued until the mid-1990s, is today one of the most widely cultivated fish species in the world. Infection with Streptococcus agalactiae is a major threat to Nile Tilapia in aquaculture around the world. This type of streptococcosis causes acute septicemia and meningo encephalitis. Antibiotic therapy is the indicated method for controlling outbreaks, preferably with the use of vaccines. Oxytetracycline, a tetracycline antibiotic with a broad spectrum of action, is the preferred antibiotic for the treatment of bacterial diseases in aquaculture, due to its relative efficacy, low $\operatorname{cost}^{2}$ and because it has the diffusion capacity in body fluids and tissues. ${ }^{3}$ The susceptibility to antibiotics obtained by the Minimum Inhibitory Concentration (MIC) of bacterial strains isolated from clinical cases can be used to classify isolates as wild-type and non-wild-type and to calculate provisional epidemiological cut-off values. ${ }^{3,4}$ Studies of experimental infections carried out in Brazil with Streptococcus agalactiae with MIC of $0.24 \mathrm{ug} / \mathrm{ml}$ treated with oral doses of $100 \mathrm{mg} / \mathrm{kg}$ of oxytetracycline every 12 hours showed $20 \%$ of mortality rates and $60 \%$ of persistence of infected animals. ${ }^{3}$ The PK/PD models relate pharmacokinetic (PK) parameters with pharmacodynamic parameters (PD) and are widely used as indicators of the efficacy of antibiotic treatments in humans and animals. These models optimize antimicrobial therapy and minimize resistance selection. ${ }^{5}$

Studies with Brazilian clinical isolates of Streptococcus agalactiae describe the frequency and distribution of MICs, ${ }^{6}$ but there are no studies evaluating the impact of these MIC changes on the clinical efficacy of oxytetracycline at usual doses. The objective of this study was to evaluate the effect of MIC changes on mortality, carrier and cure rates in Streptococcus agalactiae infections using PK/PD modeling.

\section{Materials and methods}

A PK/PD modeling was performed for the determination of the efficacy of oral oxytetracycline $100 \mathrm{mg} / \mathrm{kg}$ every 12 hours of Nile Tilapia infected with Streptococcus agalactiae strains with MICs of 0.06 ; $0.125 ; 0.25$ and $0.5 \mathrm{ug} / \mathrm{mL}$. The efficacy parameter used was the area under the plasma concentration versus time curve/minimum inhibitory concentration (AUC/MIC).$^{5-7}$ The pharmacokinetic parameters used were obtained from the pharmacokinetic study conducted by ChunYacoh et al. ${ }^{8}$ The selection of the pharmacodynamic parameters was based on data from the epidemiological study conducted by Gozi 6 with bacteria isolated from Nile Tilapia in the States of Minas Gerais, São Paulo and Parana in Brazil. Statistical analysis was performed using the Excel and BioEstat 5.0programs. In order to estimate the rates of cured, carrier and killed animals, a Monte Carlo simulation of the pharmacokinetic parameters was performed, followed by PK/PD modeling. ${ }^{5,9,10}$ The rates of cured, carrier and dead animals corresponding to each MIC were compared using the Qui-Square independence test with a significance of $\mathrm{p}=0.01$. 


\section{Results}

(Figure 1) shows the relationship between plasma oxytetracycline concentrations and MIC values. MIC values of $0.5 \mathrm{ug} / \mathrm{mL}$ were found to be close to the mean plasma concentration 3hours post administration. (Figure 1) Relation of the distributions of oxytetracycline concentrations (after oral administration of $100 \mathrm{mg} / \mathrm{kg}$ in Nile Tilapia) with MICs ofStreptococcus agalactiae (isolated from infected animals in Brazil), adapted from Chen et al. ${ }^{8}$ and Gozi. ${ }^{6}$ The $\mathrm{PK} / \mathrm{PD}$ modeling allowed to estimates the efficacy of the treatment according to the changes in the MIC of the isolated battery. Changes in estimated mortality and carrier rates related to variations in the MIC were observed (Figure 2). The estimated mortality rates were $0.3 \% ; 2.5 \% ; 25 \%$ and $90 \%$ and carrier rates were $1.3 \% ; 16.7 \% ; 60 \%$ and $9 \%$ according to the MICs of $0.06 ; 0.125 ; 0.25$ and $0.5 \mathrm{ug} / \mathrm{mL}$, respectively.

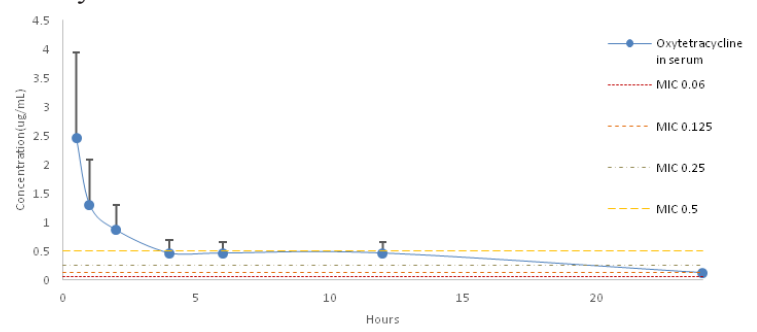

Figure I Relation of the distributions of oxytetracyc line concentrations (after oral administration of $100 \mathrm{mg} / \mathrm{kg}$ in Nile Tilapia) with MICs of Streptococcus agalactiae (isolated from infected animals in Brazil), adapted from Chen et al. ${ }^{8}$ and Gozi. ${ }^{6}$

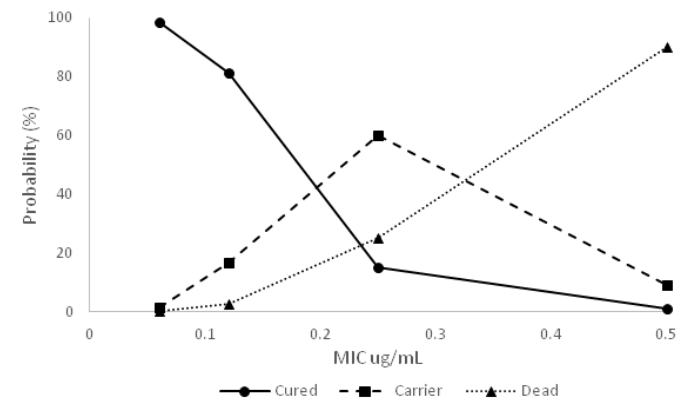

Figure 2 Probability of obtaining cured, carrier and dead animals after treatment with oral oxytetracycline $100 \mathrm{mg} / \mathrm{kg}$ for 10days in Nile Tilapias according to MIC of Streptococcus agalactiae isolated based on Monte Carlo simulation.

The probability of obtaining bacterial cure after treatment was significantly decreased for infections caused by MICs greater than $0.06 \mathrm{ug} / \mathrm{mL}(\mathrm{p}<0.001)$ (Figure 2). In the case of carrier animal rates, the probability was significantly increased for infections caused by bacteria with MICs greater than $0.06 \mathrm{ug} / \mathrm{mL}(\mathrm{p}<0,001)$. (Figure 2) Probability of obtaining cured, carrier and dead animals after treatment with oral oxytetracycline $100 \mathrm{mg} / \mathrm{kg}$ for 10 days in Nile Tilapias according to MIC of Streptococcus agalactiae isolated based on Monte Carlo simulation.

\section{Discussion}

Oxytetracycline is the main antibiotic for the treatment of clinical infections by experimental studies with clinical bacterial strains demonstrating changes in its efficacy. ${ }^{3}$ In addition; the present study demonstrates how small increases in MIC could have a high impact on therapeutic efficacy. This study demonstrates, probabilistically, that cure would be found in $80 \%$ of the animals, mortality in $2.5 \%$ and presence of carriers in $16 \%$ of the animals would be found by infection with Streptococcus agalactiae with MIC of $0.125 \mu \mathrm{g} / \mathrm{ml}$.

Gozi ${ }^{6}$ isolated 51 Streptococcus agalactiae from Nile Tilapia in 3 Brazilian states, detecting a variation of MIC to oxytetracycline of 0.2 to $1 \mu \mathrm{g} / \mathrm{mL}$ in the isolates, with a prevalence of $0.06 \mu \mathrm{g} / \mathrm{mL}$ and the epidemiological cut was set at $0.25 \mu \mathrm{g} / \mathrm{mL}$. Using this cut-off point, the present study demonstrates cure in only $15 \%$ of the animals, with death of $25 \%$ and $60 \%$ of the animals as carriers. Faria et al., ${ }^{3}$ obtained the 29 strains of Streptococcus agalactiae from isolates of tilapias in 6 Brazilian states where prevalence of strains with MIC of $0.06 \mu \mathrm{g} / \mathrm{mL}$ was $10 \%$. According to the results of the present study, oxytetracycline at doses of $100 \mathrm{mg} / \mathrm{kg}$ would only be indicated for this $10 \%$ of the cases. In addition, in the study by Faria et al., ${ }^{3}$ it was demonstrated that $80 \%$ of strains of Streptococcus agalactiae isolated had MICs between 0.125 and $0.5 \mu \mathrm{g} / \mathrm{mL}$. The present PK/PD analysis allows us to infer that the use of oxytetracycline could be used due to the susceptibility to the drug by the bacteria, but to obtain adequate efficacy results the corresponding dose adjustments should be made.

The therapeutic success of antibiotic therapy depends mainly on the relationship between the concentration of the drug at the infection site and the minimum inhibitory concentration of the bacterium causing the infection. ${ }^{5-11}$ Therefore, the therapeutic failure could be of pharmacokinetic origin (PK), pharmacodynamic (PD) or a combination of both. Therapeutic failures of PK origin occur when the drug does not reach the required concentration at the action site and those of PD origin when the bacterium resists the original therapeutic concentrations. ${ }^{11-13}$ These therapeutic failures of PD origin are evidenced by increases in pharmacodynamic parameters as minimum inhibitory concentration. ${ }^{5,14}$ In this sense, the PK/PD models correlate PK parameters with PD parameters and are widely used as indicators of the efficacy of antibiotic treatments. ${ }^{5,7,11}$ These models allow us to optimize therapeutic doses maximizing efficacy and minimizing resistance selection. ${ }^{15,16}$

These models would optimize the therapeutic doses of streptococcosis in Nile Tilapia in cases that oxytetracycline MICs are equal to or greater than $0.125 \mathrm{~g} / \mathrm{mL}$. In addition, these models allow the adjustment of therapeutic doses according to the regional changes of bacterial susceptibility to the antibiotics used, increasing the rates of therapeutic success, reducing cases of toxicity and contributing to the control of the generation of bacterial resistance.

\section{Conclusion}

In this study it was observed that small increments in the MIC determined large changes in the rates of cured, carrier and dead animals. This demonstrates the need to incorporate in the therapeutic protocol bacteriological isolation, MIC determination and optimization of therapeutic doses in order to avoid therapeutic failures and consequently potentiate the development of resistance. However, further studies are needed to determine additional pharmacokinetic parameters such as bioavailability, protein binding and dose proportionality in Nile Tilapia in order to optimize dose in the treatment of Streptococcus agalactiae diseases. 


\section{Acknowledgements}

None.

\section{Conflicts of Interest}

Author declares there is no conflict of interest.

\section{References}

1. Food and Agriculture Organization of the united nations- FAO (2013). Rome, Italy. Geneva: Fishery and aquaculture statistics FAO; 2015.

2. Rigos G, Smith P. A critical approach on pharmacokinetics, pharmacodynamics, dose optimisation and withdrawal times of oxytetracycline in aquaculture. Reviews in Aquaculture. 2015;7(2):77106.

3. Faria FC, Leal CAG, Carvalho Castro GA, et al. Carrier state induced by oxytetracycline therapy against streptococcosis in Nile tilapia, Oreochromisniloticus (L.). Journal of Fish Diseases. 2013;37(9):853-857.

4. Maisak H, Jantrakajorn S, Lukkana M. Antibacterial activity of Tannin from Seet Chestnut Wood against Aeromonas and Streptococcus pathogens of tilapia (Oreochromis niloticus). Thai J Vet Med. 2013;43:105-111.

5. Ahmad I, Huang L, Hao H, et al. Application of PK/PD Modeling in Veterinary Field: Dose Optimization and Drug Resistance Prediction. BioMed Research International. 2016;1-12.

6. Gozi KS. Antimicrobial resistance profile of Aeromonas sp. and Streptococcus sp. isolates of Nile tilapia and detection of the genes involved in resistance to tetracycline. Dissertation (Master in Aquaculture). Universidade Estadual Paulista. Jacoticabal, 2016.

7. Martinez M, Toutain PL, Bousquet MA. AUC / MIC : A PK / PD Index for Antibiotics with a Time Dimension or Simply a Dimensionless Scoring Factor ?. J Antimicrob Chemother. 2007;60(6):1185-1188.

8. Chen CY, Wooster GA, Getchell RG, et al. Distribution and depletion of oxytetracycline in two warm-water fish: tilapia and hybrid striped bass. Journal of the World Aquaculture Society. 2007;36(4):564-569.

9. Dudley MN, Ambrose PG. Pharmacodynamics in the study of drug resistance and establishing in vitro susceptibility breakpoints: ready for prime time. Current Opinion in Microbiology. 2000;3(5):515-521.

10. Trang M, Dudley M, Bhavnani SM. Use of Monte Carlo simulation and considerations for PK-PD targets to support antibacterial dose selection. Curr Opin Pharmacol. 2017;36:107-113.

11. Toutain PL. 2017. Standard PK/PD Concepts Can Be Applied to Determine a Dosage Regimen for a Macrolide: The Case of Tulathromycin in the Calf. Journal of Veterinary Pharmacology and Therapeutics. 2017;40(1):16-27.

12. Yuanan $\mathrm{H}$, Cheng $\mathrm{H}$. Health Risk fromVeterinaryAntimicrobial Use in China'sFood Animal Production and Its Reduction. Environmental Pollution. 2016;219: 993-997.

13. Torneke K, Torren edo J. The Management of Risk Arising from the Use of Antimicrobial Agents in Veterinary Medicine in EU/EEA Countries-a Review. J Vet Pharmacol Ther. 2015;38(6);519-528.

14. Chideroli RT. Emergence of a New Multidrug-Resistant and Highly Virulent Serotype of Streptococcus Agalactiae in Fish Farms from Brazil 2017. Aquaculture. 2017;479:45-51.

15. Abdelraouf K, Linder KE, Nicolau DP, et al. Predicting and Preventing Antimicrobial Resistance Utilizing Pharmacodynamics: Part I Gram Positive Bacteria. Expert Opin Drug Metab Toxicol. 2016;12(3):267280.

16. Xiong M. Relationshipbetween Cefquinome PK/PD Parameters and Emergence of Resistance of Staphylococcus Aureus in Rabbit TissueCage Infection Model. Front Microbiol. 2016;7:874. 